\title{
Clearing the Dust-Off Abiotrophia defectiva
}

\author{
Najmus Sahara, c, John S. Czachor ${ }^{\mathrm{a}}$, Mohammad Shahid ${ }^{\mathrm{b}}$
}

\begin{abstract}
Abiotrophia defectiva (ABI), a nutritional variant Streptococcus (NVS), is an uncommon cause of infective endocarditis (IE) involving both native and prosthetic heart valves. Due to the fastidious nature and special nutritional requirements, contribution of ABI to IE had been underestimated. Here we describe a case of Abiotrophia spp. native valve endocarditis in a 40-year-old female intravenous drug user who did not have any other potential source of infection. Blood cultures grew ABI along with Acinetobacter spp. perhaps from licking the needle before injecting. Transesophageal echocardiogram showed mobile vegetations attached to tricuspid and mitral valves. Susceptibility testing is important due to underlying differences in susceptibility to both penicillin and ceftriaxone between $\mathrm{ABI}$ and other genera of NVS, though both antibiotics are recommended alternate empiric first-line therapies along with synergistic gentamicin use in accordance with established guidelines to treat NVS endocarditis. She was successfully treated with intravenous ceftriaxone and gentamicin for 6 weeks with clinical and echocardiographic resolution of IE. This case highlights the importance of modern microbial techniques and equipment for accurate and rapid identification of challenging pathogens.
\end{abstract}

Keywords: Nutritional variant Streptococci; Abiotrophia defectiva; Susceptibility of Abiotrophia; Culture negative endocarditis

\section{Introduction}

Abiotrophia defectiva (ABI) is a pleomorphic, nonsporulating, non-motile, and facultative anaerobic organism that can appear as Gram-positive cocci, coccobacilli or bacilli. As a pathogen requiring specific culture media for growth, it is possible that $\mathrm{ABI}$ has been under identified in past bacterial endocarditis cases. The following is a case of infective endocarditis (IE) in a young intravenous drug user (IVDU) due to ABI. In the new era of advancement in diagnostic modalities along with availability

Manuscript submitted July 25, 2019, accepted August 5, 2019

a Division of Infectious Diseases, Wright State University Boonshoft School of Medicine, Dayton, OH, USA

bBacha Khan Medical College, Mardan, Pakistan

${ }^{\mathrm{c} C o r r e s p o n d i n g ~ A u t h o r: ~ N a j m u s ~ S a h a r, ~ D i v i s i o n ~ o f ~ I n f e c t i o u s ~ D i s e a s e s, ~}$ Wright State University Boonshoft School of Medicine, 128 East Apple Street, Second Floor, Dayton, OH 45409-2902, USA. Email: drnajum83@yahoo.com

doi: https://doi.org/10.14740/jmc3348 of susceptibility profile for some of the challenging pathogens, the complications and treatment failure rate have been improved significantly.

\section{Case Report}

A 40-year-old woman presented to the emergency department with fever, chills, and painful swollen left hand and left foot. She was an active drug user who injected heroin and methamphetamine. The patient was found on the street moaning in pain several hours after her friend injected methamphetamine into her left arm. Though she denied licking the needle before or after use, she was not sure if her friend did it. Her past medical history includes tricuspid valve endocarditis due to methicillin-sensitive Staphylococcus aureus (MSSA) 3 years prior, chronic hepatitis $\mathrm{C}$ infection, and a history of recurrent skin and soft tissue infections with abscesses. She had no drug allergies and was not on any medications at home.

Vital signs included temperature of $101^{\circ} \mathrm{F}$, pulse 130 beats per minute (bpm), blood pressure $126 / 76 \mathrm{~mm} \mathrm{Hg}$, and respiratory rate 20 breaths per minute, saturating $94 \%$ on room air. Physical examination was significant for poor dentition but no broken tooth or open sores in her mouth. She had a new grade III/IV systolic murmur along left sternal border, needle stick marks on bilateral arms, and erythematous, edematous tender hands and dorsum of the left foot. Other components of physical examination including head, eye, ear, nose, throat (HEENT), lungs, abdomen, skin and central nerves system were normal. Laboratory workup revealed normal complete blood count, basic metabolic panel, and lactic acid, though she had a mildly elevated aspartate aminotransferase (AST) at $65 \mathrm{U} / \mathrm{L}$ and total bilirubin $1.4 \mathrm{mg} / \mathrm{dL}$.

Two sets of blood cultures were obtained before empirical antibiotic therapy with piperacillin/tazobactam $3.375 \mathrm{~g}$ every $8 \mathrm{~h}$ and vancomycin $1 \mathrm{~g}$ every $12 \mathrm{~h}$ was initiated for skin and soft tissue infection, and the patient was admitted for further investigations. Both sets of aerobic blood cultures grew Abiotrophia species after 1-day incubation period, sensitive to vancomycin (minimum inhibitory concentration (MIC) $1 \mu \mathrm{g} /$ $\mathrm{mL}$ ), levofloxacin (MIC $2 \mu \mathrm{g} / \mathrm{mL}$ ), and meropenem (MIC 0.5 $\mu \mathrm{g} / \mathrm{mL}$ ). Susceptibility was intermediate to ceftriaxone (MIC 2 $\mu \mathrm{g} / \mathrm{mL}$ ), and penicillin (MIC $1 \mu \mathrm{g} / \mathrm{mL}$ ). One out of two blood culture bottles also grew Acinetobacter species, sensitive to ceftriaxone (MIC $\leq 8 \mu \mathrm{g} / \mathrm{mL}$ ), gentamicin (MIC $\leq 4 \mu \mathrm{g} / \mathrm{mL}$ ), and levofloxacin (MIC $\leq 2 \mu \mathrm{g} / \mathrm{mL}$ ).

Transesophageal echocardiogram revealed a $1.0 \times 0.5 \mathrm{~cm}$ mobile vegetation attached to tricuspid valve along with $0.5 \times$ $0.36 \mathrm{~cm}$ mobile vegetation on anterior mitral valve leaflet. Based on these findings, the diagnosis of IE was established, and was 
managed medically with intravenous antibiotics in the absence of any urgency for surgical intervention. Based on microbiology data, ceftriaxone $2 \mathrm{~g}$ daily along with gentamicin $1 \mathrm{~g} / \mathrm{kg} / \mathrm{dose}$ every $8 \mathrm{~h}$ were chosen for therapy to encompass both organisms in accordance with established guidelines for a 6-week period. Repeat blood cultures after 3 days of effective antibiotic therapy were negative. A repeat transthoracic echocardiogram after 10 days showed mild thickening of mitral valve without any gross vegetation on either valves mentioned above. During a followup visit after completing antibiotic therapy, she reported significant improvement in all of her symptoms. Repeat blood cultures 2 months after therapy remained negative.

\section{Discussion}

ABI belongs to the group of nutritional variant Streptococci (NVS), and had been included as a cause of culture negative endocarditis (CNE) in the past, secondary to difficulty in culturing this pathogen on regular culture media [1]. CNE is defined as clinical and/or echocardiographic evidence of IE, but at least three blood cultures remain negative after 7 days of incubation. Approximately $5-7 \%$ of patients with IE will have sterile blood cultures. Recent use of antibiotic therapy and infection with fastidious organisms are the most common causes of CNE (Table 1).

NVS are fastidious Gram-positive bacteria, first observed by Frenkel and Hirsch in 1961 [2]. They are part of the normal human oropharyngeal, gastrointestinal and urogenital flora, and require pyridoxal and L-cysteine supplemented media to grow. They usually grow as white-grey, non-hemolytic, satellite colonies around bacteria producing these compounds such as Staphylococcus aureus. Chromosomal DNA-DNA hybridization in 1989 and 16S rRNA gene sequencing data in 1995 and 2000 [3] led to reclassification of NVS into two genera: ABI with a single subclass as ABI defectiva, and Granulicatella (GRA) subcategories into GRA adjacens, GRA elegans, and GRA balanopterae.

A recent rise in the number of reported cases of IE due to ABI suggests improved and advanced techniques to identify this difficulty to culture pathogen by traditional methods.

Bacteremia and IE are the most frequent clinical manifestations worldwide, with an IE incidence rate of 3 - 9 cases/100,000 in developed countries, and 5-6\% of all cases of IE due to streptococcal species [1]. Preexisting cardiac disease was found in $90 \%$ of patients, while prosthetic heart valves involvement in $10 \%$ cases. A high complication rate including septic embolization in $27 \%$, neurologic complications in $20-40 \%$ including subarachnoid hemorrhage and mycotic an-
Table 1. Common Infectious Causes of Culture Negative Endocarditis

\author{
Nutritional variant Streptococci \\ Coxiella burnetii (Q fever) \\ Bartonella species \\ Chlamydia species \\ Tropheryma whipplei \\ Legionella species \\ Brucella species \\ Aspergillus species \\ Lactobacillus species
}

eurysms, and death in $17 \%$ cases have been reported with $\mathrm{ABI}$ IE [4]. ABI has also been reported as a cause of osteomyelitis, cerebral abscess, septic arthritis, and meningitis as well [5].

A retrospective study conducted over a 15-year time period $(2000$ - 2015) in Spain revealed only 12 cases of ABI/GRA IE in study institution with an incidence rate of $1.51 \%$, compared to $0.88 \%$ of Hemophilus, Aggregatibacter, Cardiobacterium, Eikenella, Kingella (HACEK) related IE, and $16.62 \%$ viridians group streptococci (VGS) during the same time period. Among these subgroups, peri-annular complications were more common in NVS group along with higher incidence of NSV IE in IVDUs $(\mathrm{P}<0.001)$. GRA IE was more common in congenital heart disease, while mitral valve involvement was more prominent with ABI IE. Between ABI and GRA, other factors including sex, prosthetic IE, aortic involvement, penicillin susceptibility and surgical treatment were comparable [6].

Susceptibility testing of ABI species is not routinely performed due to fastidious growth and special nutritional requirement. Prior studies of antimicrobial susceptibility of ABI and GRA demonstrated significant variability in their susceptibilities to penicillin and ceftriaxone which have been first-line therapies for ABI endocarditis [7-9]. Multiple studies have demonstrated that $\mathrm{ABI}$ has significantly higher sensitivity to ceftriaxone compared to penicillin, while GRA is more susceptible to the latter than the former as presented in Table 2 [7-9]. Other antibiotics with 100\% susceptibilities for both species that can be used as a potential alternate choice include meropenem, clindamycin, levofloxacin, and vancomycin [7].

The sensitivity panel of ABI in this case demonstrated intermediate sensitivity to both penicillin and ceftriaxone but complete susceptibility to vancomycin. Due to coinfection with Acinetobacter species, the patient was treated with ceftriaxone and gentamicin. In vitro sensitivity testing and clinical response do not correlate well at times, resulting in treatment

Table 2. Literature-Based Antibiotic Susceptibilities for $A B I$ and GRA

\begin{tabular}{|c|c|c|c|c|c|c|c|c|c|}
\hline \multirow{2}{*}{ Isolate } & \multicolumn{3}{|c|}{ Tuohy et al [7], susceptibility (\%) } & \multicolumn{3}{|c|}{ Alberti et al [8], susceptibility (\%) } & \multicolumn{3}{|c|}{ Patel and Marchand-Austin [9], susceptibility (\%) } \\
\hline & PNC & CTX & VANC & PNC & CTX & VANC & PNC & CTX & VANC \\
\hline $\mathrm{ABI}$ & 8 & 83 & 100 & 10.8 & 100 & 100 & 23.8 & 100 & 100 \\
\hline
\end{tabular}

PNC: penicillin; CTX: ceftriaxone; VANC: vancomycin; ABI: Abiotrophia defectiva; GRA: Granulicatella. 
failure and the need for surgical interventions [4]. The rapid clearance of bacteremia and good clinical outcome in this case confirmed the appropriate choice of antibiotic therapy alone.

Both the American Heart Association (AHA) and British Society for Antimicrobial Chemotherapy guidelines [10, 11] recommend using a synergic combination of a beta lactam and aminoglycoside for NVS IE. These guidelines have been supported by observations illustrating penicillin-streptomycin combination synergistic effect demonstrated in vitro, as well as in experimental endocarditis due to NVS [12]. The combination of a beta-lactam antibiotic plus an aminoglycoside for 4 - 6 weeks is suggested. Vancomycin for 4 - 6 weeks is alternate for penicillin allergic patients $[10,11]$. Approximately $27 \%$ of patients require prosthetic valve replacement and $50 \%$ of the patients require surgery $[5,13]$.

\section{Conclusions}

$\mathrm{ABI}$ is a rare, yet important, cause of culture negative endocarditis. Laboratory isolation of this pathogen is difficult; however modern technology has made the identification process rapid and more accurate. Though not a classic IE pathogen associated with intravenous drug use, ABI should be included in any differential for endocarditis. Empiric antibiotic choice should be based on susceptibility testing (if available) or profile data from previously published literature.

\section{Acknowledgments}

None to declare.

\section{Financial Disclosure}

None to declare.

\section{Conflict of Interest}

None to declare.

\section{Informed Consent}

Informed consent was obtained from the patient.

\section{Author Contributions}

NS is the first author; MS contributed to literature review and JSC reviewed the case for corrections and improvement.

\section{References}

1. Roberts RB, Krieger AG, Schiller NL, Gross KC. Viri- dans streptococcal endocarditis: the role of various species, including pyridoxal-dependent streptococci. Rev Infect Dis. 1979;1(6):955-966.

2. Frenkel A, Hirsch W. Spontaneous development of L forms of streptococci requiring secretions of other bacteria or sulphydryl compounds for normal growth. Nature. 1961;191:728-730.

3. Collins MD, Lawson PA. The genus Abiotrophia (Kawamura et al.) is not monophyletic: proposal of Granulicatella gen. nov., Granulicatella adiacens comb. nov., Granulicatella elegans comb. nov. and Granulicatella balaenopterae comb. nov. Int J Syst Evol Microbiol. 2000;50(Pt 1):365-369.

4. Stein DS, Nelson KE. Endocarditis due to nutritionally deficient streptococci: therapeutic dilemma. Rev Infect Dis. 1987;9(5):908-916.

5. Kiernan TJ, O'Flaherty N, Gilmore R, Ho E, Hickey M, Tolan M, Mulcahy D, et al. Abiotrophia defectiva endocarditis and associated hemophagocytic syndrome - a first case report and review of the literature. Int J Infect Dis. 2008;12(5):478-482.

6. Tellez A, Ambrosioni J, Llopis J, Pericas JM, Falces C, Almela M, Garcia de la Maria C, et al. Epidemiology, Clinical Features, and Outcome of Infective Endocarditis due to Abiotrophia Species and Granulicatella Species: Report of 76 Cases, 2000-2015. Clin Infect Dis. 2018;66(1):104-111.

7. Tuohy MJ, Procop GW, Washington JA. Antimicrobial susceptibility of Abiotrophia adiacens and Abiotrophia defectiva. Diagn Microbiol Infect Dis. 2000;38(3):189191.

8. Alberti MO, Hindler JA, Humphries RM. Antimicrobial Susceptibilities of Abiotrophia defectiva, Granulicatella adiacens, and Granulicatella elegans. Antimicrob Agents Chemother. 2015;60(3):1411-1420.

9. Samir N Patel, A Marchand-Austin. Susceptibility profile of nutritionally variant streptococci (NVS) recovered from invasive cases in Ontario, Canada. Official Journal of The Association of Medical Microbiology and Infectious Disease Canada. 2017;2(2)11-15.

10. Baddour LM, Wilson WR, Bayer AS, Fowler VG, Jr., Tleyjeh IM, Rybak MJ, Barsic B, et al. Infective endocarditis in adults: diagnosis, antimicrobial therapy, and management of complications: a scientific statement for healthcare professionals from the American Heart Association. Circulation. 2015;132(15):1435-1486.

11. Gould FK, Denning DW, Elliott TS, Foweraker J, Perry JD, Prendergast BD, Sandoe JA, et al. Guidelines for the diagnosis and antibiotic treatment of endocarditis in adults: a report of the Working Party of the British Society for Antimicrobial Chemotherapy. J Antimicrob Chemother. 2012;67(2):269-289.

12. Carey RB, Brause BD, Roberts RB. Antimicrobial therapy of vitamin B6-dependent streptococcal endocarditis. Ann Intern Med. 1977;87(2):150-154.

13. Yerebakan C, Westphal B, Skrabal C, Kaminski A, Ugurlucan M, Bomke AK, Liebold A, et al. Aortic valve endocarditis due to abiotrophia defectiva: a rare etiology. Wien Med Wochenschr. 2008;158(5-6):152-155. 